\title{
INFLUENGE DE LA QUANTITÉ DE LAIT ET DE SA COMPOSITION SUR LA CROISSANCE DU PORCELET SOUS LA MĖRE
}

\author{
E. SALMON-LEGAGNEUR et A. AUMAI'TRE \\ Avec la collaboration technique de J. Rettagliatr, Hélène Moutel. \\ et Colette Baudrillart \\ Station de Recherches sur l'Elevage des Porcins, \\ Centre national de Recherches zootechniques, Jouy-en-Josas (Seine-et-Oise)
}

\section{SOMMAIRE}

On a cherché à préciser, sur des porcelets sous la mère, l'influence de quelques caractèristiques de l'allaitement sur la croissance. A cet effet, diverses mesures effectuées au cours de $15 \mathrm{I}$ lactations de truies de race Large White furent utilisées. Elles permirent d'étudier, pour des porcelets moyens, l'évolution des quantités de lait, de matière grasse et de matière azotée ingérées au cours de l'allaitement et l'influence de leurs variations sur la vitesse de croissance et l'indice de consommation.

On note, en particulier, que le gain journalier augmente avec les quantités consommées, mais que l'indice de consommation augmente avec l'âge et diminue avec les quantités consommées. L'effet de la composition du lait est surtout sensible pendant les premières semaines; la teneur en matière azotée paraît le facteur prépondérant.

\section{INTRODUCTION}

Durant les 4 premières semaines qui suivent sa naissance, le porcelet se nourrit à peu près exclusivement du lait de sa mère. La quantité d'éléments nutritifs qu'il ingère pendant ce temps lui permet un développement important, et son poids initial se trouve alors multiplié environ par 5 .

Plusieurs auteurs ont montré la liaison étroite qui existait entre la quantité totale de lait consommée et le poids de la portée au sevrage. C'est ainsi que nous avions trouvé un coefficient de corrélation $r=+0,857(n=7 \mathrm{x})$ entre ces deux variables à 8 semaines (SALMON-I,EGAGNEUR, I958) et AlLEN et LASLEY (I960) un coefficient de corrélation $r=+0,58(n=45)$ à 6 semaines. Toutefois, BERGE et INDREBO (I953) ont montré qu'il existait des variations dans l'utilisation du lait au cours de l'allaitement. 
En fait, on ne dispose que de très peu d'informations sur la façon dont varie la croissance d'un porcelet en fonction de la quantité et de la composition du lait consommé aux différentes époques de l'allaitement. L'étude expérimentale d'un tel phénomène est en effet très délicate, car on n'est pas maître, en général, des facteurs des variations.

Nous arons donc essayé dans un premier temps d'aborder ce problème dans les conditions naturelles (porcelets laissés en groupe sous la mère) (1).

L'objectif de ce travail est forcément limité, car les techniques utilisées ne permettent pas une étude de détail des phénomènes et, notamment, tous les facteurs individuels ont dî être laissés de côté.

Il s'agissait avant tout d'essayer de confirmer et de regrouper, à partir d'un nombre important de données (I53 lactations), les observations faites par quelques auteurs sur différents aspects de la croissance des porcelets allaités. Nous avons voulu ensuite dégager les points pour les juels une étude ultérieure plus précise serait nécessaire.

Nous avons cherché à connaître, tout d'abord, l'évolution des quantités de lait reçues par porcelet au cours des différentes semaines et la liaison entre le gain moven journalier du porcelet et ces quantités. Parallèlement, l'utilisation du lait a été suivie à l'aide de la variation de l'indice de consommation exprimé en kg de lait par $\mathrm{kg}$ de gain de poids de la portée.

En outre, dans un travail antérieur (SAIMON-LEGAGNiLR), I958, nous avions pu montrer que la production laitière des truies variait avec la taille des portées. I1 était alors intéressant de voir comment variait, dans ce cas, la part de chaque porcelet et si l'utilisation de ce lait était elle-même affectée par le nombre de porcelets au sein d'une même portée.

Enfin, Kozixfr (I950) avait observé cule les porcelets tétant les mamelles inguinales de la truie et recevant, de ce fait, un lait moins riche, notamment en matière grasse, croissaient moins vite que ceux recevant le lait des mamelles pectorales. Ceci posait le problème de l'influence de la composition du lait sur la croissance des porcelets. Nous avons alors cherché à préciser cette influence en étudiant les relations qui liaient les quantités de matière grasse et de matière azotée reçues à la croissance pondérale moyenne des animaux d'une même portée.

\section{MA'TÉRIEL F'T MÉTHODES}

\section{I - ANimaux}

Les mesures effectuées au cours de 5.3 lactations de 85 truies Large White de notre troupeau expérimental ont scrvi de matériel de départ a cette étude. Ces lactations avaient lieu dans une porcherie dont la température et l'état hygrométrique étaient maintenus constants ( $18^{\circ} \mathrm{C} ; 60 \mathrm{p}$. I00) après tme gestation pissée en plein air.

Les lactations duraient 8 sumaines pendant lesquelles toutes les truies recevaient à volonté un même aliment éfuilibré. les porcelets recevaient dins une loge à part un aliment complémentaire sous forme granulée.

(1) Nous nous proposons, par la suite, de repremoire res différents points dans une étucle plus détaillée des métabolisnes individuels sur animanx in cages de digrestibilité. 


\section{2 - MESURES DES QUANTITÉS MOYENNES DE LAIT, DE MATIÈRE GRASSE ET D'AZOTE CONSOMMÉES PAR PORCELET}

La mesure de la production laitière des truies étaient effectuée chaque semaine à l'aide de la technique que nous avons précédemment décrite (SALMON-LEGAGNEUR, 1956). A partir des courbes de lactations ainsi dressées, on déterminait graphiquenent par planimétrie, compte tenu des variations d'effectifs, les quantités hebdomadaires de lait consommées par chaque porcelet. Ces quantités étaient exprimées en $\mathrm{kg}$ de lait par porcelet et par semaine de I à 8 semaines. Il s'agit donc ici de résultats moyens obtenus par calcul et, en aucun cas, il n'a été procédé directement à la mesure de la consommation individuelle des porcelets.

72 lactations comportaient en outre une récolte d'échantillons de lait et l'analyse hebdomadaire de la composition du lait pour l'azote et la matière grasse. Ces échantillons étaient prélevé, et analysés suivant des techniques que nous avons précédemment décrites (SALvoN-LEGAGNEURI $959 a, b)$. Compte tenu des quantités de lait produites, il était ainsi possible de connaitre les quantités de matière grasse et de matière azotée ingérées par somaine par charque porcelet.

\section{3 - Détermination DE LA CROISSANCE DES PORCELETS. INDICE DE CONSOMMATION}

La croissance pondérale des porcelets était suivie à l'aide de pesées individuelle bihebdomadaires. Ces pesées permettaient de calculer le gain moyen journalier par animal et par semaine, celui-ci était exprimé en grammes. Line courbe de croissance hebdomadaire moyenne, en fonction du temps, a ainsi été tracée.

Les variations de l'utilisation du lait étaient suivies grâce à l'indice de consommation moyen, Celui-ci était exprimé en $\mathrm{kg}$ de lait par $\mathrm{kg}$ de gain de la porté au cours de chacune des $8 \mathrm{~s}$ semaines de la lactation, en tenant compte des quantités consommées par les porcelets morts en cours d'allaitement.

\section{4 - MÉTHOdés DE CALCUL}

Nous avons tout d'abord calculé les coefficients de corrélation entre les quantités de lait, les quantités de matière grasse et les quantités de matière azotée et la croissance pondérale journalière unoyenne des animaux.

Les coefficients de corrélations partielles entre ces dernières variables, calculées indépendamment des quantités de lait moyennes reçues, ont permis d'apprécier l'effet des constituants du lait (azote et matière grasse) sur la croissance du porcelet.

L'influence du nombre de porcelets sur l'utilisation du lait était déterminée par le calcul des variances et de la signification des différences entre les indices de consommation, de 5 groupes inégaux de portées de 6 à to porcelets.

\section{RÉSULTATS}

\section{I - ÉVOLUTION DES QUANTITÉS CONSOMMÉES PAR PORCEIAT \\ AU COURS DE L'ALLAITEMENT}

\section{A. - Quantités de lait}

Les quantités hebdomadaires moyennes de lait consommées par portée et par porcelet sont consignées dans le tableau I. La figure $\mathrm{I}$ montre l'évolution des quantités en fonction du temps. Il convient de noter que l'ingestion maximum se situe durant la $5^{\text {e }}$ semaine, donc semble-t-il, ne coïncide pas tout à fait avec le maximum de production de la truie, ce qui est dû à la diminution de l'effectif des portées à mesure que la lactation avance. 


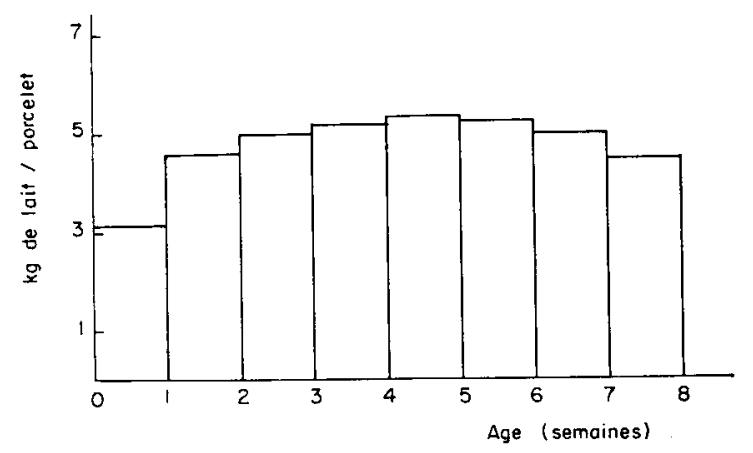

FIG. I. - Quantité de lait de triie consonmée par porcelet par semaine.

TABLEAU I

Évolution hebdomadaire de la production laitière des truies et de la quantité de lait consommée par porcelet ( $k g$ par semaine) $(\mathrm{n}=15 \mathrm{I})$

\begin{tabular}{|c|c|c|c|c|c|c|c|c|c|c|}
\hline $\begin{array}{l}\text { Age de la portée en } \\
\text { semaines } \ldots . . .\end{array}$ & 1 & 2 & 3 & 4 & 5 & 6 & 7 & 8 & 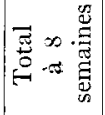 & 家芯兽 \\
\hline $\begin{array}{c}\text { Lait consommé par } \\
\text { portée }(\mathrm{kg}) \ldots \ldots\end{array}$ & $\begin{array}{r}27,78 \\
+\quad 9,74\end{array}$ & $\begin{array}{r}38,39 \\
+12,02\end{array}$ & $\begin{array}{r} \\
11,72 \\
+\quad 11,9\end{array}$ & $\begin{array}{r}42,59 \\
+10,53\end{array}$ & $\begin{array}{r}12,90 \\
\pm 10,60\end{array}$ & $\begin{array}{r}41,74 \\
+10,70\end{array}$ & $\begin{array}{r}39,20 \\
\pm 10,1\end{array}$ & $\begin{array}{r}3 \mathbf{3}, 50 \\
\pm 10,60\end{array}$ & 309,26 & 38,66 \\
\hline $\begin{array}{l}\text { Lait consommá par } \\
\text { porcelet }(\mathrm{kg}) \ldots \ldots\end{array}$ & $\begin{array}{r}3,16 \\
\pm \quad 1,13\end{array}$ & $\begin{array}{r}4,57 \\
\pm \quad 1,29\end{array}$ & $\begin{array}{r}5,00 \\
+\quad 1,30\end{array}$ & $\begin{array}{l}5,18 \\
1,23\end{array}$ & $\begin{array}{r}5,32 \\
+\quad 1,27\end{array}$ & $\begin{array}{r}5,22 \\
+\quad 1,31\end{array}$ & $\begin{array}{r}4,98 \\
上 \quad 1,35\end{array}$ & $\begin{array}{r}4,43 \\
\pm \quad 1,61\end{array}$ & 37,86 & 4,73 \\
\hline
\end{tabular}

De plus, les quantités de lait consommées par porcelet varient très peu de la $3^{\mathrm{e}}$ à $1 \mathrm{a} 6^{\mathrm{e}}$ semaine, alors qu'il convient de rappeler que les besoins des porcelets augmentent considérablement pendant la même période.

Enfin, la quantité consommée par porcelet durant la $8^{\mathrm{e}}$ semaine est encore très élevée et notablement plus importante que celle consommée pendant la première semaine de l'allaitement (4o p. Ioo de plus).

\section{B. - Quantités de matière grasse et de matière azotée}

Les quantités moyennes de matière grasse et de matière azotée (exprimée en protéines du lait $\mathrm{N} \times 6,38$ ) consommées par porcelet sont rapportées au tableau 2 et à la figure 2 .

L'examen des courbes de la figure 2 montre que la quantité de matière grasse consommée par porcelet augmente jusqu'à la $4^{\mathrm{e}}$ semaine, puis diminue. Par contre, la quantité de matière azotée consommée est maximum à la $6^{\mathrm{e}}$ semaine, puis diminue lentement durant les 2 dernières semaines de lactation. 
TABLEAU 2

Eíolution des quantitl's de matière grasse et de matière asolie hebdomadaires ingivées par porcelet $\left(\mathrm{n}=\tau_{2}\right)(\mathrm{g})$
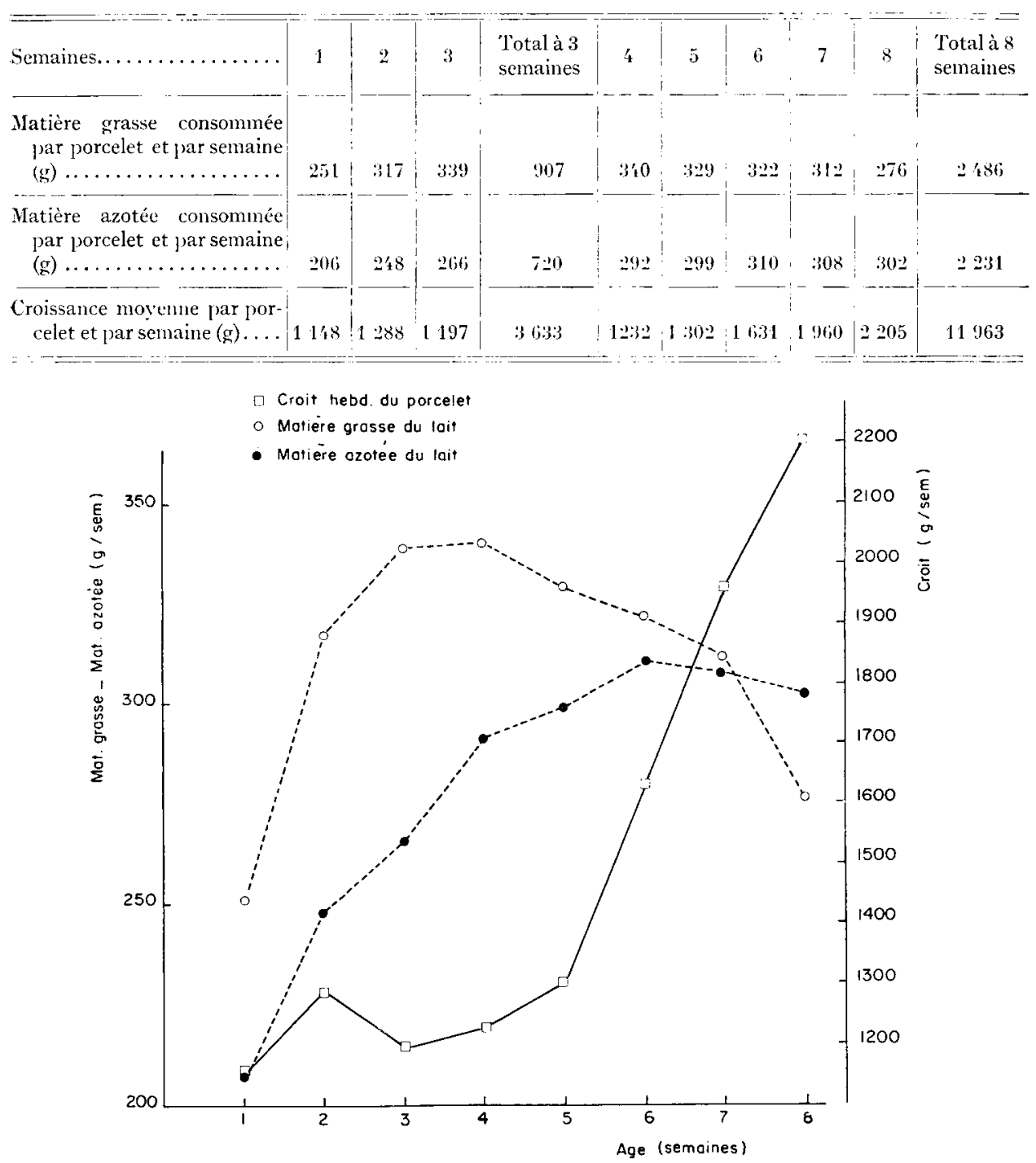

FiG. 2. - Evolution comparée des quantités de matière asotée el de matière grasse du lait consommées et de la croissance des porcelets.

2 - INFLUENCE DE I,A QUANTITÉ DE LAIT CONSOMMÉE PAR PORCELET

A. - Sur la croissance pondérale

a) Phénomène global :

Cette influence apparaît dans les coefficients de corrélation entre la croissance et les quantités de lait reçues par porcelet que nous rapportons au tableau 3 . 
TABLEAU 3

Coefficients de corrélation éntre la croissance à 3 et à 8 semaines et les quantités de lait correspondantes $(\mathrm{n}=15 \mathrm{I})$

\begin{tabular}{|c|c|c|}
\hline & $r$ & $\begin{array}{l}\text { Seuil de signi- } \\
\text { fication }\end{array}$ \\
\hline $\begin{array}{l}\text { Quantité de lait consommée par porcelet à } 3 \\
\text { semaines et croissance } 0 \text { à } 3 \text { semaines ...... } \\
\text { Quantité de lait consommée par porcelet à } 8 \\
\text { semaines et croissance } 0 \text { à } 8 \text { semaines ....... }\end{array}$ & $\begin{array}{l}+0,870 \\
+\quad 0,530\end{array}$ & $\begin{array}{l}\mathrm{P}<0,01 \\
\mathrm{P}<0,01\end{array}$ \\
\hline
\end{tabular}

Comme on pouvait le supposer, il existe une corrélation étroite entre le gain pondéral du porcelet pendant 1'allaitement et la quantité de lait correspondante. On remarque toutefois que le coefficient de corrélation est plus faible pour toute la période de l'allaitement (o à 8 semaines), que de o à 3 semaines. Ceci peut s'expliquer par le fait que le porcelet consomme dès la $5^{\mathrm{e}}$ semaine une quantité non négligeable d'aliment complémentaire (Aumaitre et SALMON-L,EGaGNEur, Ig6I).

b) Evolution au cours de la lactation :

I es coefficients de corrélation pour chaque semaine entre la croissance et la quantité de lait consommée par porcelet sont rapportées au tableau 4 .

\section{TABLEAU 4}

Coefficient de corrélation entre la croissance myyenne et la quantité de lait consommé par porcelet chaque semaine $(\mathrm{n}=\mathrm{I} 5)$

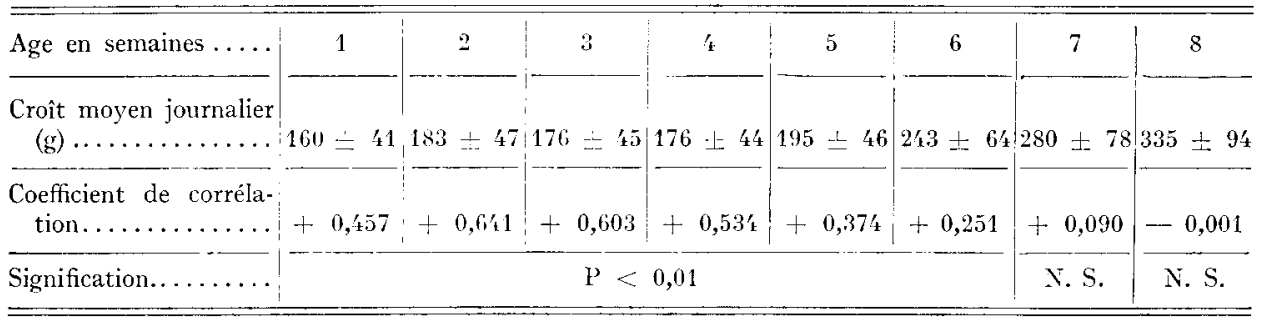

On remarque que l'influence du lait sur la croissance diminue avec l'âge de la portée. A partir de la $7^{\mathrm{e}}$ semaine, une augmentation de la quantité de lait reçue par animal ne semble pas influer sur la croissance.

\section{B. - Sur l'indice de consommation}

a) Variation avec la quantité totale de lait consommée :

L,es indices de consommations à 4 et 8 semaines, ainsi que les coefficients de corrélation entre la quantité de lait consommée et ces indices sont rapportées dans le tableau 5 . 


\section{TABLEAU 5}

(uantiti's de lait par porcelet $(\mathrm{kg})$ - indice de insommation (l. (C) et coefficients de corrélation entre les 2 irariables

\begin{tabular}{|c|c|c|c|c|c|}
\hline Age & $\begin{array}{l}\text { Nombre de por- } \\
\text { celets par portée }\end{array}$ & $\begin{array}{c}\text { Quantité mov- } \\
\text { enne de lait par } \\
\text { porcelet }\end{array}$ & $\begin{array}{l}\text { I. C. mosen kgr } \\
\text { lait/kgrain }\end{array}$ & $\begin{array}{l}\text { Coefficient de } \\
\text { corrélation totale }\end{array}$ & Signification \\
\hline 't semaines .... & $8, x^{\prime}= \pm 1,88$ & $17,9 \div 3,9$ & $3,80=0, f(0)$ & $+0,225$ & $P<0,01$ \\
\hline 8 semaines ..... & $8,08 \pm 1,98$ & $37,7 \pm 7,5$ & $3,52=0,58$ & $+0,430$ & $P<0,01$ \\
\hline
\end{tabular}

La valeur des coefficients de corrélation indique que l'indice de consommation augmente légèrement avec la quantité de lait (le phénomène est d'ailleurs plus net à 8 semaines qu'à 4 semaines). Ce qui revient à dire que le porcelet utilise moins bien le lait lorsqu'il en obtient beaucoup.

b) I'ariation avec l'âge du porcelet:

Les valeurs de l'indice de consommation, calculées semaine par semaine sont rapportées au tableau 6. Comme l'avait déjà montré BERGE (r953), cet indice de consommation augmente avec 1'âge durant la phase lactée exclusive. A partir de la $5^{\mathrm{e}}$ semaine, on observe toutefois une diminution qui peut être due à l'ingestion d'aliment complémentaire.

$$
\text { TABIEAU } 6
$$

Érolution de l'indice de consomination moyen au cours des semaines surcessives

\begin{tabular}{|c|c|c|c|c|c|c|c|c|}
\hline Semaines .......... & 1 & 2 & 3 & it & 5 & 6 & 7 & 8 \\
\hline I. C. $(\mathrm{kg})$ & 2,86 & 3,66 & 4,22 & 4,39 & 4,27 & $3,2 \geq$ & 2,74 & 2,14 \\
\hline
\end{tabular}

\section{c) Influence du numéro de portée de la truie :}

Le tableau 7 révèle de très faibles différences dans les indices de consommation selon le numéro d'ordre de la portée. L'utilisation du lait serait notamment un peu meilleure au cours de la première portée que des suivantes. Toutefois, le calcul du test $\mathrm{F}$ montre que ces différences ne sont pas significatives.

TABLEAU 7

Indice de consommation et numéro d'ordre de portée de la truie

\begin{tabular}{|c|c|c|c|c|c|c|c|c|}
\hline Numéro d'ordre de la portée .... & 1 & 2 & 3 & & 禾 & & 5 & $6,7,8,9$ \\
\hline Nombre d'observations ....... & 35 & 33 & 27 & & 17 & 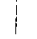 & 18 & 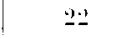 \\
\hline $\begin{array}{l}\text { Indice de consommation }(0 \text { à } 8 \text { se- } \\
\text { maines }) \ldots \ldots \ldots \ldots \ldots \ldots \ldots\end{array}$ & 3,56 & 3,80 & 3,86 & 1 & 3,80 & & 3,70 & 3,81 \\
\hline
\end{tabular}




\section{3 - INFLUENCE DU NOMBRE DE PORCELETS AU SEIN DES PORTÉES}

\section{A. - Sur la quantité de lait consommée par porcelet}

Lorsque le nombre de porcelets d'une portée augmente, la quantité de lait consommée par chaque porcelet diminue, comme le montre le tableau 8 .

\section{TABLEAU 8}

Variation de la quantité de lait consonnie par porcelet ayec la taille de la portée

\begin{tabular}{|c|c|c|c|c|c|}
\hline Effectif de la portée...$\ldots \ldots \ldots$ & $3-1$ & $5-6$ & $7-8$ & $9-10$ & $11-12$ \\
\hline Nombre d'observations........... & 6 & 27 & 57 & 49 & 13 \\
\hline $\begin{array}{l}\text { Quantité de lait consommée par } \\
\text { porcelet }(\mathrm{kg}) \ldots \ldots \ldots \ldots \ldots \ldots\end{array}$ & 55,8 & 42,4 & 37,6 & 35,6 & 33,7 \\
\hline
\end{tabular}

On peut montrer de la même façon que, quel que soit le stade de lactation, on trouve toujours une corrélation négative entre la quantité de lait consommée par porcelet et l'effectif de la portée (tableau 9).

\section{TABLEAU 9}

Ézolution des coefficients de corrélation entre la quantité de lait consommée par porcelet et le nombre de porcelets

\begin{tabular}{|c|c|c|c|c|c|c|c|c|}
\hline $\begin{array}{l}\text { Age de la portée en se- } \\
\text { maines } \ldots \ldots \ldots \ldots\end{array}$ & 1 & 2 & 3 & 4 & 5 & 6 & 7 & 8 \\
\hline$r \ldots \ldots \ldots \ldots \ldots \ldots$ & $-0,330$ & $-0,293$ & $-0,3 \div 6$ & $-0,422$ & $-0,414$ & $-0,363$ & $-0,387$ & $-0,433$ \\
\hline Signification......... & & & & $\mathrm{P}<$ & 0,01 & & & \\
\hline
\end{tabular}

a) Gain journalier:

\section{B. - Sur la croissance}

Le tableau ro rapporte les valeurs des coefficients de corrélation totale

TABLEAU IO

Éolution des coefficients de corrólation entre la croissance et le nombre de porcelets

\begin{tabular}{|c|c|c|c|c|c|c|c|c|}
\hline $\begin{array}{l}\text { Age de la portée en se- } \\
\text { maines } \ldots \ldots \ldots \ldots\end{array}$ & 1 & 2 & 3 & 3 & 5 & 6 & 7 & 8 \\
\hline $\begin{array}{c}\text { Croissance/nombre de } \\
\text { porcelets au sevrage }\end{array}$ & $-0,215$ & $-0,087$ & $-0,276$ & $-0,135$ & $+0,057$ & $+0,034$ & $+0,061$ & $\rightarrow 0,001$ \\
\hline Signification ........ & $\mathrm{P}<0,05$ & N. S & $P<0,05$ & X. S & N. S & N.S & N. S & N. S \\
\hline $\begin{array}{r}\text { Croissance/nombre de } \\
\text { porcelet (quantité de } \\
\text { lait par porcelet cons- } \\
\text { tante) } . \ldots \ldots \ldots \ldots \ldots\end{array}$ & $-0,076$ & $-0,375$ & $-0,090$ & $+0,117$ & $-0,239$ & $+0,139$ & $+0,105$ & $+0,001$ \\
\hline Signification & N. $S$ & $\mathrm{P}<0,01$ & N. $S$ & N. $S$ & $\mathrm{P}<0,01$ & N. S & N. S & N. S \\
\hline
\end{tabular}


et partielle (quantité de lait constante) liant le nombre de porcelets et le gain journalier moyen aux différents stades.

On constate que le gain journalier des porcelets n'est que très peu affecté par la variation de l'effectif de la portée. Comme parallèlement les quantités de lait consommées diminuent, on peut penser ' ju'il en résulte une meilleure utilisation du lait par les animaux.

b) Indice de consommation :

Nous avons trouvé, en effet, un coefficient de corrélation totale négatif $(r=-$ $0,4 \mathrm{II}, \mathrm{P}<\mathrm{O}, \mathrm{OI}$ ) entre l'indice de consommation et le nombre de porcelets au sevrage. Ceci apparait aussi au tableau in ou nous rapportons la variation de cet indice de consommation en fonction du nombre de porcelets au sevrage.

TABIEAU II

Indie de ionsommation obsere'e sur des porties de taille rariable

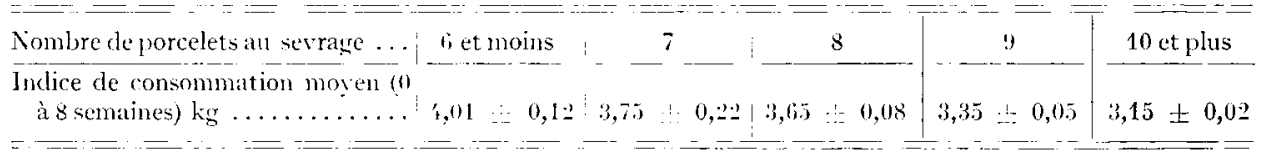

L'analyse de variance $(F=5,98, P<0,0 I)$ montre que les différences observées sont significatives.

Il semble donc que, dans les limites de notre étude, l'utilisation du lait par les porcelets soit d'autant meilleure que l'effectif de la portée est plus élevé. Indirectement, nous trouvons ici une confirmation des résultats du tableau 5 , à savoir que le rendement de l'utilisation du lait par les porcelets diminue lorsque la quantité de lait augmente.

'Toutefois, il semble qu'il y ait aussi une influence directe de l'effectif de la portée, car la corrélation négative entre l'indice de consommation et le nombre de porcelets persiste à quantité de lait consommée constante. On trouve, ell effet, dans ce cas :

$$
\begin{array}{ll}
r=-0,246 & \mathrm{I}^{\prime}<0, \mathrm{OI} \text { à } 4 \text { semaines, } \\
r=-0,244 & \mathrm{I}^{\prime}<0, \mathrm{O} \text { à } \mathrm{S} \text { semaines. }
\end{array}
$$

\section{4 - INFluence de la COMposition dU LAIT SUR IA CROISSANCE}

A. - Influence des quantités totales de matière grasse et de matière azotée

Cette influence peut s'apprécier à l'aide des coefficients de corrélation à 3 semaines ou à 8 semaines, que nous rapportons au tableau $\mathbf{2}$.

T.ABI,EAC I 2

Comrilations éntre le's quantitis de' matière grasse, de matiere acolté du lait et la croissance des porcelels

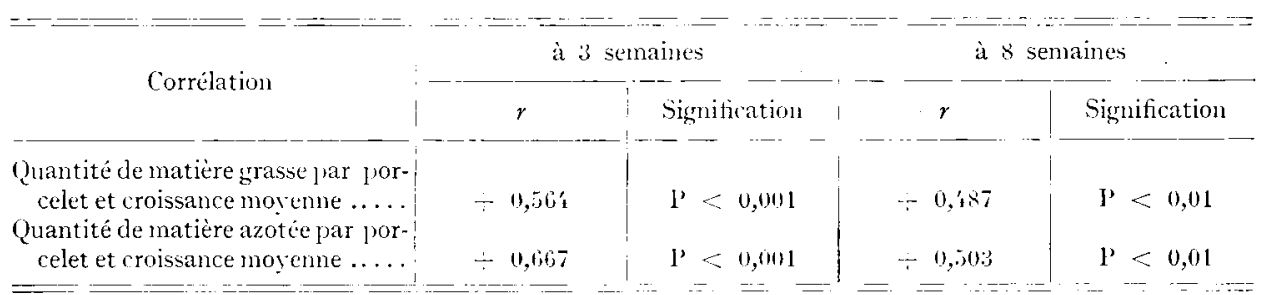

Amnales de Zootechnie. - I, ()$_{2}$. 
On constate que la vitesse de croissance du porcelet angmente lorsque la quantité de matière grasse ou de matière azotée ingérée par ce dernier s'accroît.

\section{B. - Erolution au cours de l'allaitement}

I es tableaux I3 et I4 rapportent l'évolution des coefficients de corrélation entre les quantités hebdomadaires de matière azotée ou de matière grasse du lait ingérées et la croissance correspondante.

\section{TABI.EAT I3}

Coefficients de conrélation entre la quantite' de mative grasse on de maticire azotée consommite et la croissance invern.

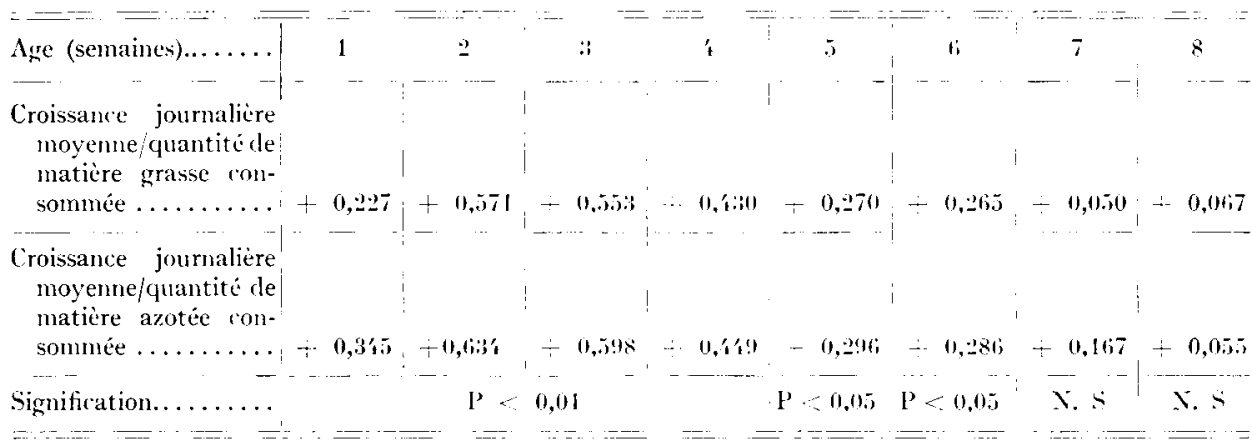

TABIEAL It

Coeffrients de corrélation partielle entre les quantite's de matiere grasse. les quantitis de matiere asotie et la croissante

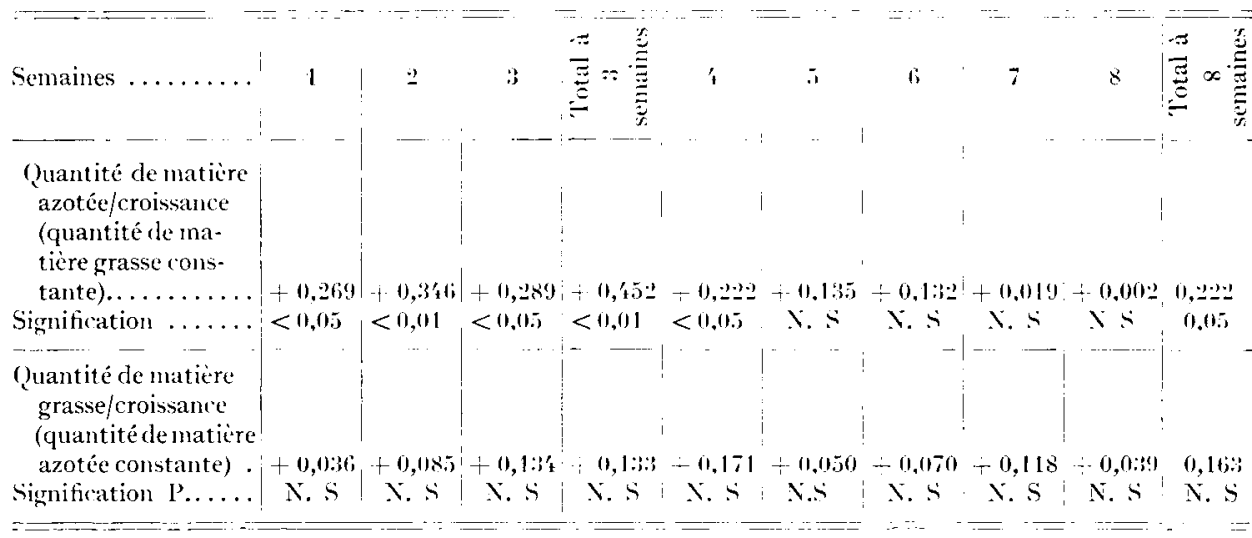

On remarque, dans le tableau I3, que la liaison entre ces différentes variables est élevée et significative pendant les 6 premières semaines. Elle est maximale à la deuxième semaine et diminue ensuite. Nous retrouvons ici le même phénomène que pour les quantités de lait.

Par contre, lorsqu'il s'agit de corrélations partielles (indépendantes de l'un des facteurs), les valeurs trouvées sont beaucoup plus faibles et ne sont significatives que dans le cas de la matière azotée. 


\section{DISCUSSION}

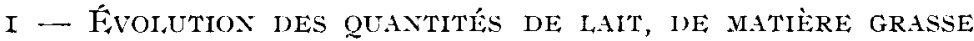 ET DE MATIÈRE AZOTÉE COXSOMMÉES}

I)ans notre étude, les quantités de lait consommées par porcelet sont tout à fait comparables à celles indiquées par BERGE et INidREBo (I953) et suivent la même évolution. Comme ces auteurs en effet, nous avons trouvé que la quantité maximum ingérée par porcelet se situait aux environs de la $5^{\mathrm{e}}$ semaine (tableau $\mathrm{I}$ ).

Mais il est assez étonnant de constater que la quantité de lait hebdomadaire reçue par chaque porcelet varie fort peu, alors que le gain journalier est susceptible de varier plus largement (tableaux $I$ et 4 ). Ceci ne fait que traduire la part croissante de l'ingestion d'aliment complémentaire dans ce gain journalier.

Le fait qu'au sevrage, à $\delta$ semaines, la quantité de lait qu'obtient le porcelet soit encore relativement élevée, montre que cette époque ne correspond pas forcément avec celle de l'arrêt physiologique de la sécrétion du lait chez la truie.

L'évolution des quantités de matière grasse et de matière azotée consommées appelle peu de commentaires. On peut souligner, toutefois, que la quantité de matière azotées ingérées augmente pratiquement jusqu'au sevrage, alors que la quantité de matière grasse baisse assez fortement à partir de la $4^{\mathrm{e}}$ semaine. Il s'en suit que dès cette époque, le rapport azote énergie de l'ingéré a tendance à s'élever alors que l'on s'accorde à reconnaitre que le rapport des besoins suit l'évolution inverse (Cunha, I957). Ce déséquilibre a pour conséquence que, passé un mois, le lait n'est peut-être plus l'aliment idéal du jeune porcelet et que l'utilisation du lait à ce moment est peut-être moins bonne.

\section{2 - INFLUEATCE DES QUANTITÉS DE IAIT SUR IA CROISSANCE}

\section{A. - Gain de poids}

De nombreux auteurs ont trouvé un coefficient de corrélation élevée entre le poids de la portée au sevrage et la production laitière de la truie (Boxsya, I935; SAlmox-Legagreur, I958 ; Suith, I959 $a$; Lodge et MAC IONAI.d, I959 $a$; AlleN ET LASLEY, I960). Les coefficients de corrélation que nous avons trouvés entre la croissance des porcelets et la quantité moyenne de lait reçue confirment ces résultats et montrent donc la part importante de l'ingestion du lait dans la croissance du jeune.

Mais l'évolution de ces coefficients de corrélation au cours de l'allaitement paraît plus intéressante à considérer. Comme BERGE (I953), en effet, nous avons trouvé que la liaison entre le lait et la croissance était très étroite pendant chacune des quatre premières semaines, mais qu'elle diminuait très fortement ensuite. OWEN (I957) a aussi observé sur des agneaux allaités que le coefficient de corrélation liant la croissance et le lait ingéré diminuait très fortement de la $2^{\mathrm{e}}$ à la $S^{\mathrm{e}}$ semaine d'allaitement. 
On peut trouver une double explication à ce phénomène :

$\left.I^{0}\right)$ Les besoins du porcelet augmentent très rapidement et l'apport de lait qui est à peu près constant, ne couvre plus qu'une partie de ces besoins. En effet, pour une même quantité de lait, la fraction utilisée pour l'entretien s'accroît avec le développement de l'animal, alors que celle utilisée pour la croissance diminue;

$\left.2^{\circ}\right)$ Par contre, l'alimentation complémentaire joue un rôle de plus en plus important et fait qu'à partir de la $6^{\mathrm{e}}$ semaine notamment, les quantités relativement élevées de lait encore ingérées sont de peu d'effet sur la croissance en regard des aliments complémentaires (AUMAITRE et SALMON-I,EGAGNEUR, I96I).

Ce dernier point attire l'attention sur le moment exact auquel il convient de procéder au sevrage. Si l'on s'en tient aux quantités journalières de lait ingérées, il est tout aussi arbitraire de sevrer le porcelet à 8 semaines qu'à 6 semaines puisque ces quantités diffèrent peu. Si l'on se base sur l'utilisation du lait, le sevrage à 6 semaines paraît intéressant puisque dès cette époque la part du lait dans la croissance devient très faible et qu'il y a donc gaspillage. Ceci n'est possible, toutefois, que dans la mesure où l'apport de lait ne revêt pas un caractère indispensable poutr le porcelet, ce qui semble admis à la lumière des expériences sur les régimes synthéti(ues (CusHA, I957).

\section{B. - Indice de consommation}

La première chose à souligner est l'excellente utilisation du lait maternel qui est faite par le porcelet lorsqu'on la compare à celle d'autres espèces domestiques de grande taille. I)ans notre travail, dont les résultats confirment pleinement ceux de BERGE (r953), nous avons trouvé un rendement moyen, ou indice de consommation, de la naissance à un mois, de $\mathrm{I} \mathrm{kg}$ de croît pour $3,84 \mathrm{~kg}$ de lait de truie.

Chez l'agneau, qui présente des caractéristiques d'allaitement assez voisines de celles du porcelet (même gain de poids, lait de composition analogue), le rendement est nettement moins bon. C'est ainsi que RicordeAu. et Boccard (I96I) trouvent chez l'agneau Préalpe un indice de consommation compris entre 5,33 et $6,08 \mathrm{~kg}$ de lait par $\mathrm{kg}$ de croît pendant les 6 premières semaines.

Chez le veau, toute proportion gardée, l'indice de consonmation est encore plus élevé (il est voisin de ro), mais le lait ingéré a une composition assez différente des deux premières espèces. De telles variations traduisent en effet les différences de composition et de valeur nutritionnelle des laits, les différences du poids à la naissance et de la composition des tissus de croissance et probablement une caractéristique de croissance de l'espèce.

Nos résultats montrent ensuite que l'indice de consommation augmente très sensiblement lorsque la quantité de lait ingéré croît. Ce phénomène, qu'avait également indiqué I ONAI,D (I937) semble assez général, puisque OWEN (I957) le signale aussi chez l'agneau. Il est vraisemblable qu'une partie de l'explication provient aussi de la nature des tissus de croissance. Selon le schéma bien connu de Mc MEekar (I94I), lorsque l'ingéré est élevé, l'animal en consacre tune partie importante à l'élaboration de tissus adipeux, cô̂teux en énergie, ce qui diminue le rendement pondéral de la croissance.

Le fait que l'indice de consommation augmente avec l'âge du porcelet (tableau 6) relève sans doute du même phénomène, mais indique également, comme nous le disions plus haut, que la part du besoin d'entretien s'accroît au détriment de la 
croissance. Il peut également être une conséquence de l'évolution que nous avons signalée du rapport azote/énergie du lait consommé.

Enfin, la légère augmentation de l'indice de consommation avec le numéro d'ordre de la portée, ou ce qui revient au même, avec 1'âge des truies, provient sans doute de ce que la quantité de lait produite est plus grande au cours des $2^{\mathrm{e}}$ et $3^{\mathrm{e}}$ portée que de la I $^{\mathrm{re}}$ (SALMON-LEGAGNeur, I958).

\section{3 - INFLUENCE DU NOMBRE DE PORCELETS}

La première conséquence de l'augmentation du nombre de porcelets élevés au sein d'une portée est la diminution de la quantité de lait qui revient à chacun. De ce fait, l'incidence sur la croissance est donc la suivante : ralentissement de la vitesse de croissance et baisse de l'indice de consommation. Mais si l'influence sur la vitesse de croissance est relativement modérée (tableau ro), les variations de l'indice de consommation sont assez sensibles. C'est ainsi que cet indice baisse d'environ 20 p. roo lorsque le nombre de porcelet passe de 6 à Io.

Ce phénomène est d'ailleurs assez général, puisqu'on le retrouve dans d'autres espèces. C'est ainsi que Schurze (1954) sur la Ratte, OWEN (1957) et Ricordeau et BoCCARD (I96I) sur la Brebis ont montré que, lorsque le nombre de petits augmentait, le lait maternel était mienx utilisé.

Ceci pose alors le problème du nombre optimum de jeunes à conserver au sein d'une portée pour provoquer la croissance totale la plus importante. Retenons tout d'abord que des jeunes en nombre élevé sollicitent et obtiennent au total davantage de lait de la mère que s'ils sont peu nombreux (SCHUlzE, I954; BERGE, I953 ; SAIMON-LEGAGNEUR, I958). Comme par ailleurs l'utilisation individuelle du lait est également meilleure lorsque l'effectif est important, il semble donc indiqué, du moins théoriquement, de conserver un nombre élevé de petits sous la mère. Notre étude, toutefois, ne nous donne pas de renseignements lorsque l'effectif excède I2 porcelets. Il est vraisemblable, en effet, compte tenu des difficultés d'allaitement que l'on rencontre alors, que ce dernier nombre corresponde à un maximum. D'ailleurs, la croissance totale et l'utilisation du lait ne constituent pas les seuls critères permettant de définir la taille optima de la portée.

\section{4 - INFLUENCE DE T,A COMPOSITION DU LAIT}

L'influence de la matière grasse et de la matière azotée du lait sur la croissance du jeune pendant la période lactée est beaucoup plus délicate à interpréter. KozinER (I950) avait jobservé une croissance plus élevée des porcelets tétant les 4 mamelles antérieures de la truie qui produisent un lait plus riche en matière grasse. Cependant, on s'accorde à dire que la production de lait de ces mamelles est nettement plus élevée que celles des mamelles postérieures (DONALD, I937; BARBER et BRAUde, I955; SAIMON-LEGAGNeUR, I958) et il n'est pas certain que l'influence respective de la quantité de lait et du taux de matière grasse sur la croissance ait été dissociée dans l'étude de KozINER (I950). On peut faire la même critique au travail de GUTTE et LENKEIT (I956), qui montrent qu'un apport azoté plus important provoque une meilleure croissance des porcelets. Dans ce cas aussi il y avait aug- 
mentation de la quantité totale du lait et notamment d'énergie. De même, GLAwisCHNIG (I96I) a observé une diminution de la croissance des porcelets pendant la $3^{\text {e }}$ semaine correspondant à une diminution de $I \$ \mathrm{p}$. Ioo du taux de matière azotée du lait de la mère, cependant l'étude portait sur un nombre très faible d'animaux et là aussi d'autres facteurs ont pu intervenir.

Nos propres travaux n'infirment aucune de ces hypothèses, car nous avons trouvé des corrélations également élevées entre les quantités de matières grasses et de matière azotée du lait et la croissance des jeunes animaux. Toutefois, il n'est pas inutile de souligner que la valeur de ces corrélations est beaucoup plus faible lorsque celles-ci sont calculées de façon indépendante (tableau I 4), c'est-à-dire à quantité de matière grasse constante pour l'azote et à quantité d'azote constante pour la matière grasse. Par exemple, à 3 semaines, les valeurs respectives des deux coefficients pour la matière azotée et la matière grasse sont respectivement de $+0,45$ et $-0,13$. Ceci semble indiquer que l'action de ces deux constituants du lait est cumulative et que c'est en fin de compte la quantité totale de nutriments ingérés (autrement dit la quantité de lait) qui a le rôle principal. Mais, il convient surtout de remarquer que la corrélation est un peu plus élevée (et significative) pour l'azote, ce qui confère à ce facteur une place plus importante que celle de la matière grasse.

Par ailleurs, nos résultats sont en désaccord avec ceux de PEO et al. (I957) qui ont trouvé avec des porcelets alimentés avec un régime artificiel à base de lait écrémé en poudre, une liaison inverse, non significative, entre le taux de matière grasse (apporté sous forme de saindoux) et la croissance du porcelet. Ces auteurs, ont, par contre, observé une amélioration de la croissance avec des taux élevés de protéines. Je son côté, Simith (I959 b) observe pour des quantités de matière grasse de lait de truie sensiblement égales ( $3500 \mathrm{~g}$ et $3700 \mathrm{~g})$ et des rapports matière grasse matière azotée respectifs de $I, 63$ et $\mathrm{I}, 47$, une diminution de $\mathrm{I} \mathrm{kg}$ du poids moven des porcelets recevant moins de matière azotée.

En fait, il paraît très difficile de séparer les influences respectives de l'azote et de la matière grasse, puisque ces deux facteurs varient en général dans le même sens, qui est aussi celui de la (quantité totale de lait (SAIMON-LEGAGNEUR, Ig6I). En outre, si le calcul des coefficients de corrélation partielle (tableau I4) semble indiquer que durant les 3 premières semaines de la vie du jeune, la matière azotée joue un róle prépondérant par rapport à la matière grasse, il n'en est plus ainsi ensuite. I es mêmes coefficients à 8 semaines montrent, en effet, que l'influence de l'un et l'autre nutriment sur la croissance est faible et qu'en définitive aucun d'eux n'a un effet important à ce stade.

\section{CONCLUSION}

Comme on aura pu le constater, les facteurs qui font varier l'utilisation du lait maternel par porcelet sont très nombreux. Leur étude est, en outre, rendue complexe par le fait que la plupart de ces facteurs sont liés entre eux.

Nous avons esssayé dans ce travail de préciser la part qui revient à quelquesuns d'entre eux, et on retiendra surtout les points suivants : 
a) La croissance est liée avant tout à la quantité de lait ingérée jusqu'à 6 semaines. La liaison est beaucoup moins nette ensuite. Ceci peut poser le problème d'un sevrage éventuel des porcelets à cet âge. L'une manière plus générale, le coefficient d'utilisation du lait de truie par le porcelet décroît avec l'âge et avec la quantité ingérée par ce dernier.

b) Le nombre de porcelets d'une portée a une influence sensible sur la quantité de lait consommée par chaque porcelet et l'utilisation de ce lait. L'utilisation du lait paraît en effet meilleure lorsque l'effectif est élevé. Comme par ailleurs la vitesse de croissance est peu affectée, il s'en suit que les portées nombreuses semblent permettre la meilleure valorisation des aptitudes laitières des truies.

c) L'influence de la composition du lait sur la croissance est délicate à dégager, car elle a donné lieu à des conclusions parfois contradictoires. Cette influence se manifeste surtout pendant les premières semaines de l'allaitement. La matière azotée et la matière grasse ingérées sont en relation avec la croissance. Le rôle le plus important semble dévolu à l'ingéré protidique, mais des études plus poussées seront nécessaires pour préciser l'influence relative exacte de chacun de ces deux constituants de la sécrétion lactée.

$$
\text { Recu pour publication en mai } 1962 .
$$

\section{SUMMARY}

THE INFI, LAXE OF THE MILK CONSUMPTION AND COMPOSITION UPON THE GRONTH OF SUCKLING P'IGLETS.

I53 lactations of 85 sows have been used to study the influence of milk consumption on the growth of piglets. In this study, the weekly amount of milk, nitrogen matter and fat received by the piglets were taken into account. During the lactation, the feed efficiency of milk ( $\mathrm{kg}$ milk/ $\mathrm{kg}$ piglet) varies from 2,9 (ist week) to 4,4 (4th week) and the correlation beetwen daily gain and milk varies from : $+0,64$ (2d week) to $+0,09$ (7th week).

The feed efficiency increases with the number of piglets in the litter and decreases with the age of piglets and the amount of milk eaten. The influence of milk composition is mostly during the first 4 weeks and seems more important for nitrogen matter than for fat (partial correlation at 3 weeks : $+0,45$ versus $+0,13)$.

Conclusions are drawn about the interest of weaning piglets towards $5^{-6}$ weeks

\section{RÉFÉRENCES BIBLIOGRAPHIQUES}

Allen A. D., Iasley J. F., 1960. Nilk production of sows. J. Anim. Sci., 19, 150-355.

Aumaitre A., Salmon-Legagnelr E., ig6r. Influence de l'alimentation complémentaire sur la croissance du porcelet avant le sevrage. Ann. Zootech., 10, i 26-I 40.

Barber R. S., Bratide R., Mrtchell K. G., 195. Studies on milk production of Large-White pigs. J.Agric., Sci., 46, 97-I I8.

Berge S., Ivorebo T., I953. Mjolkeproduksjon hos purker. Meld. Norg. LandbHogsk., 73, 390-424.

Bonsma F.N., Oosthuizex P. M., 1935. Milk production in Large-Black sows. S. A1r.J.Sci., 32, $360-378$.

Braude R., Townsenil J. M., Rowel S. G., ig60. A comparison of meal and pelleted forms of creep for suckling pigs. J. Agric. Sci., 54, $274-27 \%$.

Cunin J. J., I957. Swine feeding and nutrition. Interscience publishers, N. Y., 36-39.

Donald H. P., I937. The milk consumption and growth of suckling pigs. J. Exp. Agri., 5, 349-359.

Glawischnig F., 196I. Zum Einfluss des Gehaltes an tierischen Eiweiss im Futter der trächtigen oder der der laktierenden Sau auf dem Hamoglobinwert der Saugferkels. Wien. Tierärstl. Mschr, 4, $249^{-2} 5^{8 .}$ 
Gutte J.O., Rachal l'., Lexkert W., ig56. Litersuchungen Zum N-ansatz von Saugferkels und dessen und dessen Abhändigkeit von der liweissaufnahme. \%. Tieremahr., 11, 259-322.

Koziner A. B., i95o. Teneur en maticre grasse du lait de truie et croissance des porcelets sous lat mere (Russe). Sotzial Zhivalnoz., 12, 96-98.

LEROY A. M., LERY (i., 1946. Expériences sur lat croissance des porcelets, pendant la période d'allaitement et de sevragre. Inn, agron., 1, 1-1 7 .

L.ODGE G. A., Mac DONAI.D I., I959 a. The relative intluence of brith weight, milk consumption and supplementary food consumption upon the growth rates of suckling piglets. Inim. Prot., 1, I39-144.

JODGE G. A., $1959 \mathrm{~b}$. The energy requirement of lactating sow and the inlluence of level of food intake upon milk production and reproductive cycle. J. Agric. Sci., 53, I77-191.

MCMeekan C. P., I9H. Growth and development in the pig, with special reference to carcass quality characters. J.Agr. Sici., 31, $\mathrm{x}-49$.

Owen J. B., I957. A study of the lactation and growth of hill sheep in their native environuement and under lowland conditions. J. Agric. Sci., 48, $38 \%-4 \mathrm{I}$.

Ricordeay G., Boccand R., r96r. Relations entre la quantité de lait comsonméte par les agneaux et leur croissance. Ann. Zootech., 10, 113-125.

I'eo E. R., Ashton G. C., Speer V.C., Catrox 1.. V., 1957. Protein and fat requirement of haby pigs. J. Anim. Sci., 16, $885-892$.

Salmon-Legarneur E., I956. La mesure de la production laitière chez la truie. Inn. Zootech., 5, 95-I Io.

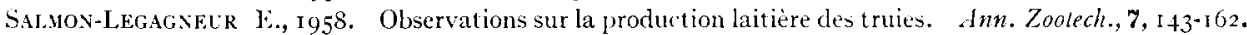

SALMon-Legagneli E., I959 a. La composition du lait de truie : prenieres observations sur quelques facteurs de variation. Ann. Zootech., 8, 93-112.

Salmon-Legagiejr E., 1959 b. Description et utilisation diune nachine a traire les truies. . Inn. Zoolech., 8, $345 \cdot 352$.

Samon-Leganier F., ig6r. La composition du lait de truie. Relation entre les variations des teneurs du lactose et des autres constituants. Ann. Biol. anim. Bioch. Biophy's., 1, 205-303.

SCIILLTEE M. O., I95-. Weight increment of suckling rats as affected by litter size and maternal diet. $J$. Nitir., 54, 453-460.

Sinth D. M., r959 a. The yield and composition of milk from sows fed varying proportions of separated milk and concentrates. N. Z. J. Agr. Res., 2, 1057-10;0.

SuIth D. M., r959 b. The yield and composition of milk from sows fed on three ration levels. $N . Z$. J. Agr. Res., 2, $107 \mathrm{I}-1083$. 